\title{
Geometrical effects in antiproton annihilation on nuclei
}

\author{
J. Cugnon \\ Université de Liège, Institut de Physique B5, Sart Tilman, B-4000 Liège 1, Belgium \\ S. Wycech \\ Sołtan Institute for Nuclear Studies, Hoża 69, PL-00-681 Warsaw, Poland \\ J. Jastrzȩbski and P. Lubiński \\ Heavy Ion Laboratory, Warsaw University, PL-02-097 Warsaw, Poland
}

(Received 22 September 2000; published 23 January 2001)

\begin{abstract}
The importance of geometrical effects in the $A$ dependence of many observables regarding antiproton annihilation on nuclei is expected on theoretical grounds. The cases of the charged pion multiplicity and of the frequency of the so-called single nucleon events are examined. In the first case, the respective roles of surface diffuseness, partial nuclear opacity, and pion absorption mechanism are examined. The possibility of extracting from comparison with experiment the average location of the annihilation site is critically discussed. The obtained values are in agreement with current theoretical estimates.
\end{abstract}

DOI: 10.1103/PhysRevC.63.027301

Annihilation of stopped or low-energy antiprotons on nuclei presumably takes place close to the nuclear surface. It is therefore expected that many observables are predominantly influenced by simple geometrical aspects. As a matter of fact, it was predicted on theoretical grounds [1] that many quantities relative to antiproton annihilation at rest should show a smooth $A$ dependence, which takes a simple form when expressed in term of $\Omega$, the solid angle under which the target is seen from the (average) annihilation point. Yet, few attempts have been made to extract from the $A$ dependence the location of the average annihilation point and consequently, to check the common belief expressed above. The first work in that direction is due to Polster et al. [2]. Using basically a strong absorption model with a sharp nuclear surface, these authors expressed the charged pion multiplicity as a linear function of $\Omega$ and extracted the location of the average annihilation point. They found that the distance $\delta$ separating this point from the half-density radius sphere is $\sim 1.1$ fm. In this paper, we reanalyze the existing data and properly take account of two neglected features, partial transparency and nuclear surface diffuseness. We also investigate the role of the pion absorption mechanism. Furthermore, we apply the same considerations to a subset of annihilation events, namely, the so-called single nucleon events. In the latter, the target is left with only one nucleon missing and with a very low excitation energy (basically below neutron emission threshold). It is generally accepted that in these events pions issued from the annihilation are just "missing" the target nucleus on their way out of the annihilation site. The authors of Refs. [3-5] were indeed able to show that the relative probability of these events is given by the so-called missing probability (for pions), which depends upon the location of the annihilation process. We show below that in this case, the value of $\delta$, extracted from experiments [3-5], is substantially larger than the one coming from the analysis of pion multiplicities for all events. We argue that this originates from the fact that the two experiments involve different modes of interaction.
PACS number(s): 25.43. $+\mathrm{t}$

Let us assume, as in Ref. [2], that annihilation (at rest) occurs at an average distance $x_{0}=R+\delta, R$ being the half density radius, from the center of the nucleus and that $N_{\pi}^{\text {prim }}$ (primordial) pions are emitted from this point at random directions. Due to pion absorption by the target nucleus, a smaller number $N_{\pi}$ of pions survive:

$$
\left\langle N_{\pi}\right\rangle=\left\langle N_{\pi}^{\mathrm{prim}}\right\rangle P
$$

where $P$ is the average survival probability for the emitted pions. Assuming that these pions travel along straight line trajectories and are provided with a constant absorption cross section $\sigma_{\text {abs }}$, the probability $P$ can be put in the following form:

$$
P=\frac{1}{4 \pi} \int d \Omega \exp \left[-\int_{0}^{\infty} d z^{\prime} \sigma_{\text {abs }} \rho\left(z^{\prime}\right)\right],
$$

where $z^{\prime}$ is the coordinate along any direction issued from the annihilation point (see Fig. 1) and where $\rho$ is the nuclear density. It is quite often argued that pions are absorbed on two nucleons [6-8]. Therefore the following expression for $P$ :

$$
P=\frac{1}{4 \pi} \int d \Omega \exp \left[-\int_{0}^{\infty} d z^{\prime} \frac{\sigma^{\prime}}{\rho_{c}}\left[\rho\left(z^{\prime}\right)\right]^{2}\right],
$$

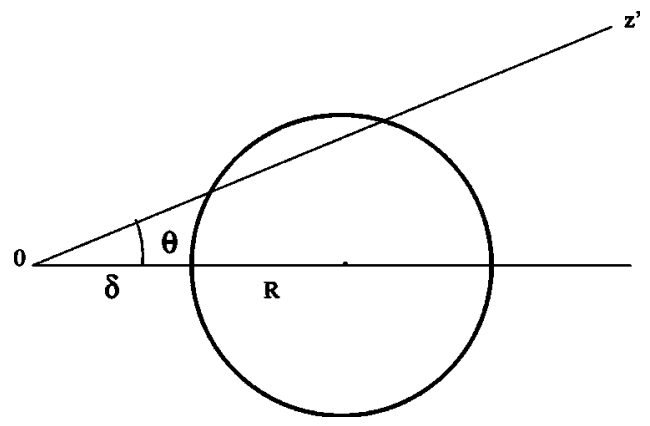

FIG. 1. Definition of the geometrical variables used in this paper. $R$ is the half-density nuclear radius. 
TABLE I. Best fit values for pion multiplicity data.

\begin{tabular}{lccccc}
\hline \hline & & \multicolumn{2}{c}{ First absorption model } & \multicolumn{2}{c}{ Second absorption model } \\
\hline Global fit & $\begin{array}{l}\text { Best fit } \\
\text { values } \\
\chi^{2} / N_{\mathrm{DF}}\end{array}$ & $\sigma=16 \pm 2 \mathrm{mb}$ & $\delta=1.60 \pm 0.1 \mathrm{fm}$ & $\sigma=42 \pm 4 \mathrm{mb}$ & $\delta=1.35 \pm 0.1 \mathrm{fm}$ \\
& & 0.75 & 1.15 \\
\hline $\begin{array}{l}\text { Fit of pion-nucleus } \\
\text { absorption data }\end{array}$ & $\begin{array}{c}\text { Best fit } \\
\text { values } \\
\chi^{2}\end{array}$ & $\sigma=16 \mathrm{mb}$ & & $\sigma=42 \mathrm{mb}$ & \\
\hline $\begin{array}{l}\text { Fit of antiproton } \\
\text { data only }\end{array}$ & $\begin{array}{c}\text { Best fit } \\
\text { values } \\
\chi^{2} / N_{\mathrm{DF}}\end{array}$ & $\sigma=44 \mathrm{mb}$ & $\delta=2.7 \mathrm{fm}$ & $\sigma=75 \mathrm{mb}$ & $\delta=2.0 \mathrm{fm}$ \\
\hline \hline
\end{tabular}

where $\rho_{c}$ is the nucleon density at the center of the nucleus, is also plausible. The absorption coefficient is written in such a way as to introduce the quantity $\sigma^{\prime}$ that has the dimension of a surface and that can be compared to $\sigma_{\text {abs }}$. In fact, expressions (2) and (3) are equivalent for a nucleus with a uniform density $\rho\left(z^{\prime}\right)=\rho$ and a sharp surface (provided $\sigma^{\prime}$ $\left.=\sigma_{\text {abs }}\right)$ and then become

$$
\begin{aligned}
P= & \frac{1}{2}\left\{1+\cos \theta_{0}+\int_{\cos \theta_{0}}^{1} d(\cos \theta)\right. \\
& \left.\times \exp \left[-2 \rho \sigma_{\mathrm{abs}} R\left(1-\frac{x_{0}^{2}}{R^{2}} \sin ^{2} \theta\right)^{1 / 2}\right]\right\},
\end{aligned}
$$

where $\theta_{0}$ is the opening angle of the cone tangent to the nuclear surface with the apex on the annihilation point. In the strong absorption limit $\left(\sigma_{\mathrm{abs}} \rightarrow \infty\right)$, this formula reduces to

$$
P=1-\frac{\Omega}{4 \pi}
$$

with

$$
\frac{\Omega}{4 \pi}=\frac{1}{2}\left\{1-\left[1-\left(\frac{R}{R+\delta}\right)^{2}\right]^{1 / 2}\right\} .
$$

In Ref. [2], the experimental data (referring to charged pions) are described by a linear form in $(1-\Omega / 4 \pi)$, that is, however, slightly different from Eqs. (1),(5),(6):

$$
\frac{\left\langle N_{\pi}\right\rangle}{\left\langle N_{\pi}^{\text {prim }}\right\rangle}=a\left(1-\frac{\Omega}{4 \pi}\right)+b,
$$

with $a=1.33$ and $b=-0.22$. This result is obtained, with a constant $\delta=1.15 \mathrm{fm}$, from data ranging from $H$ to ${ }^{238} \mathrm{U}$. However, this analysis is not satisfactory for two reasons. First, formula (2) of Ref. [2] for the solid angle $\Omega$ is an approximation of the correct expression [Eq. (6)], only valid for smaller values of $\delta / R$ than those involved here. Second, the fit does not correspond to the right limit when $\Omega \rightarrow 0$. The authors of Ref. [2] require that $\left\langle N_{\pi}\right\rangle /\left\langle N_{\pi}^{\text {prim }}\right\rangle$ be unity for the annihilation on a proton, which they associate with $1-\Omega / 4 \pi$ $\approx 0.9$, considering $R$ as the (target) proton radius. This is a too naive interpretation of formulas (1) and (5), as for the proton case there is no remaining part of the target that can interact with the pions. It is more reasonable to remove the $H$ data.

We reanalyzed the data of Ref. [2] and those of Refs. $[6,9]$, fitting them with formulas (2) or (3) and taking $\rho(r)$ as a Woods-Saxon form. ${ }^{1}$ Giving no prejudice to either of these formulas and to the underlying models for pion absorption, we first just looked for the possibility of good fits. In both cases, we found a minimum $\chi^{2}$, corresponding to unreasonably large values of $\delta$ and $\sigma_{\text {abs }}$ (or $\sigma^{\prime}$ ) in comparison with theoretical estimates $[1,6]$, see Table I. Both best fits are roughly equivalent. However, in each case, we found a whole series of correlated values of $\delta$ and $\sigma_{\text {abs }}$ ( or $\sigma^{\prime}$ ), giving $\chi^{2}$ values close to the minimum one. This is easy to understand, as an increase (decrease) of $\delta$ can be somehow compensated by an increase (decrease) of the absorption cross section. Trying to remove the ambiguity, we also performed a fit of the pion-nucleus absorption data of Refs. [12,13] for an incident kinetic energy of $200 \mathrm{MeV}$ (the average value for pions produced in the annihilation) with similar formulas as Eqs. (2) and (3), namely,

$$
\sigma_{\mathrm{abs}}^{\mathrm{tot}}=2 \pi \int_{0}^{\infty} b d b \exp \left[-\int_{-\infty}^{+\infty} d z \rho(z) \sigma_{\mathrm{abs}}\right]
$$

and

$$
\sigma_{\mathrm{abs}}^{\mathrm{tot}}=2 \pi \int_{0}^{\infty} b d b \exp \left[-\int_{-\infty}^{+\infty} d z \frac{\sigma^{\prime}}{\rho_{c}}[\rho(z)]^{2}\right],
$$

respectively. In these equations, $b$ is the impact parameter and $z$ is the longitudinal coordinate for the given value of $b$. In practice, as the data of Refs. $[12,13]$ are rather scarce and scattered, we fitted on the parametrized $\sigma_{\text {tot }}^{\text {abs }}=\sigma A^{c}$ form provided in these references for positive pions, corrected for Coulomb distortion. The best fit is obtained for $\sigma_{\text {abs }}=16 \mathrm{mb}$ with Eq. (8) and $\sigma^{\prime}=42 \mathrm{mb}$ with Eq. (9), with roughly equivalent and rather well-defined minimum $\chi^{2}$ values (here the values of $\chi^{2}$ are calculated after providing, in the parametrized form, each value of A between 12 and 240 with an

\footnotetext{
${ }^{1}$ We used $R=r_{0} A^{1 / 3}-0.48$ fm with $r_{0}=1.18 \mathrm{fm}[10]$ and $\left\langle N_{\pi^{ \pm}}^{\mathrm{prim}}\right\rangle$ $=3.10$, corresponding to the average over $\bar{p} p$ and $\bar{p} n$ data [11].
} 


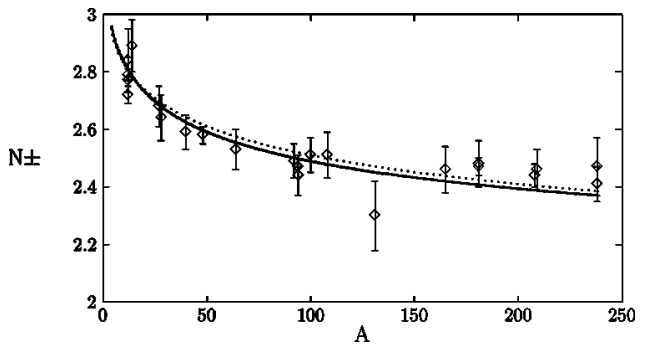

FIG. 2. Average observed charged pion multiplicity $(N \pm)$ in antiproton-nucleus annihilation at rest. The lozenges represent the data of Refs. $[2,6,9]$. The full and dotted lines give the best fits with Eqs. (3) and (2), respectively. The average number of primordial pions is taken as 3.10 , the mean value of $\bar{p} p$ and $\bar{p} n$ data [11].

error bar of about the same size as the experimental error bars). The larger value of $\sigma^{\prime}$ is understandable as the role of the surface is considerably reduced in Eq. (9). It turns out that these values are rather different from the best values we obtained by fitting the antiproton data alone. Nevertheless, it was possible to obtain a good description of both the antiproton and the pion-nucleus absorption data by keeping the same values of $\sigma_{\text {abs }}$ and $\sigma^{\prime}$ and varying the parameter $\delta$, using either $\rho$ or $\rho^{2}$ absorption. The results of these fits are given in Table I. One can see that the $\chi^{2}$ per degree of freedom for the fit of the antiproton data is only slightly larger for the global fit than for fitting these data only.

Figure 2 gives an idea of the quality of the last fit for the antiproton data. Both absorption models describe the data equally well. Figure 3 displays the results of the fit when the data are plotted, as in Ref. [2], against the variable $1-\Omega / 4 \pi$. In both absorption models, the best fit yields values close to the strong absorption limit with a sharp surface. This originates from the compensation between the diffuse surface effects, which enhance the absorption and the partial transparency of the nuclear volume which decreases it. The two effects are, however, much larger in the one-nucleon absorption model than in the two-nucleon absorption model. This statement is supported by the results of Fig. 3, where the thin lines represent the values obtained with Eq. (4), which, compared to expressions (2) and (3), somehow removes the diffuse surface effects, leaving partial transparency effects only.

The description with the first absorption model is slightly better, but the difference is so small that none of the models can be ruled out. Unfortunately, the two descriptions yield different values of $\delta$. If, however, the second model is selected, as it should perhaps be in view of its sounder theoretical foundation, one finds $\delta=1.35 \pm 0.1 \mathrm{fm}$, which is $\sim 0.2$ $\mathrm{fm}$ larger than the value extracted by Polster et al. [2]. This value is barely consistent with the theoretical values quoted in Ref. [1], namely, $\delta=1.55 \mathrm{fm}$ on the average, with values ranging from $1.44 \mathrm{fm}$ for $\mathrm{Ca}$ to $1.75 \mathrm{fm}$ for $\mathrm{Pb}$.

This result puts a strong constraint on the theoretical models for the annihilation site, which have still a limited reliability, due to uncertainties on the antiproton optical potential and on the annihilation range (see a discussion about this point in the conclusion of Ref. [1]). The correct location of the annihilation site is also important for the proper description of the subsequent pion cascades [6].

We want to apply similar considerations to this subset of
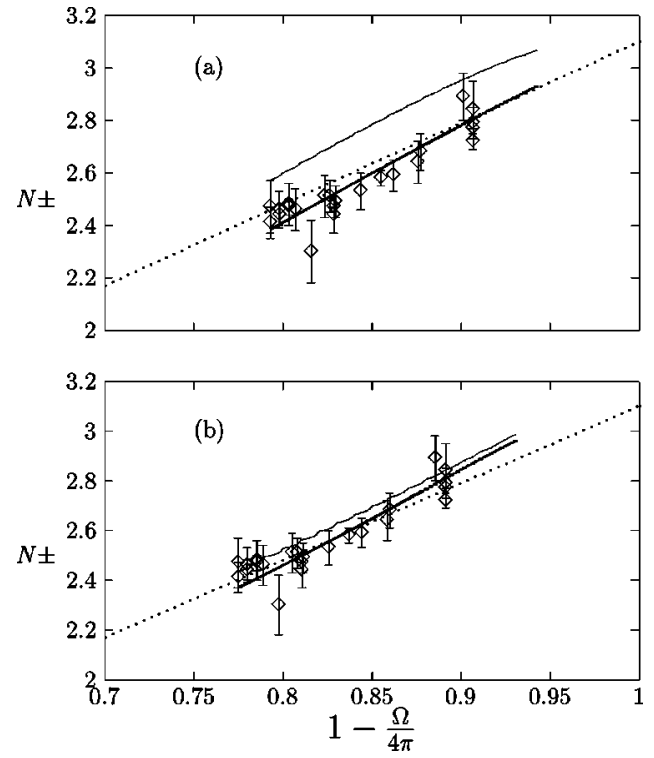

FIG. 3. Average observed charged pion multiplicity $(N \pm)$ plotted against the variable $1-\Omega / 4 \pi$ [Eq. (6)], compared with the best fits (thick lines) using Eq. (2) (upper panel) or Eq. (3) (lower panel). The dotted lines gives the strong absorption limit with a sharp surface [Eqs. (1),(5),(6)]. The thin lines represent the results of Eq. (4) with the same parameters. In each panel, the abscissa [Eq. (6)] is evaluated using the value of $\delta$ provided by the corresponding fit. The data are the same as in Fig. 2.

annihilation events. Assuming isotropic and independent emission of $k$ pions from the annihilation point, the probability of a single nucleon event, i.e., for no pion interaction (the so-called missing probability), can be given by

$$
P_{\text {miss }}=P^{k}=\left\{\frac{1}{4 \pi} \int d \Omega \exp \left[-\int_{0}^{\infty} d z^{\prime} \rho\left(z^{\prime}\right) \sigma_{\pi N}^{\text {tot }}\right]\right\}^{k} .
$$

The quantity in the curly bracket $(P)$ is similar to the one of Eq. (2), but where $\sigma_{\text {abs }}$ has been replaced by $\sigma_{\pi N}^{\text {tot }}$, the total pion-nucleon cross section. The quantity $P_{\text {miss }}$ depends upon the (average) location of the annihilation point for this particular class of events $[3,4,14,15]$. We have to average over the number of pions. Let $\omega_{k}$ be the probability of having $k$ primordial pions. One has

$$
P_{\text {miss }}=\sum_{k=2}^{k_{\max }} \omega_{k} P^{k}
$$

Of course, pions are emitted at directions which are correlated by momentum conservation. Here, we want to take account of these correlations in a simple, heuristic, way. ${ }^{2}$ The most obvious consequence of these correlations holds for the case of a sharp surface with a very large interaction cross section: if pions are emitted from the surface $(P$ $=1 / 2$ ), at least one of them is bound to cross the nucleus. Therefore the missing probability should vanish. On the other hand, expression (10) should be correct for a larger and

\footnotetext{
${ }^{2}$ It can easily be seen that such correlations do not affect the (inclusive) pion absorption probability discussed earlier.
} 
larger number of pions, as momentum conservation correlations are then less and less important. The following expression meets these two requirements:

$$
P_{\text {miss }}=\sum_{k=2}^{k_{\max }} \omega_{k} P^{k}\left[1-\left(\frac{1-P}{P}\right)^{k-1}\right] .
$$

It can be motivated as follows: to miss the nucleus [in the sharp surface plus strong interaction limit, see Eq. (5)], the $k$ pions have to be emitted outside the opening solid angle $\Omega$, without $k-1$ of them being emitted in the opposite solid angle. Of course, this does not exhaust all the possibilities for missing the nucleus, but can be considered as quite illustrative. It turns out that, for the relevant values of $P(0.65-$ $0.75)$, momentum conservation correlations are of minor importance, bringing an effect of a few percent.

Experimentally $P_{\text {miss }}$, the relative frequency of the single nucleon events compared to all annihilation events, is of the order of 10-15\% [3-5], for target mass $A$ ranging from $\sim 60$ to $\sim 200$, with perhaps a slight overall decrease with increasing $A$. We fitted the data with the help of Eqs. (10) and (12) and a Gaussian distribution for the $\omega_{k}$ 's, with a mean of 5.06 and a standard deviation of 0.84 . This distribution describes the $N \bar{N}$ experimental data [11] adequately. The best fit is given in Fig. 4. It yields $\sigma_{\pi N}^{\text {tot }} \approx 140 \mathrm{mb}$, which is a reasonable value for (isospin) average total cross section, and a value of $\delta=2.05 \pm 0.10 \mathrm{fm}$, which agrees more or less with the current theoretical estimates $[14,16,17]$ : from $\sim 2.0 \mathrm{fm}$ for $N d$ to $\sim 2.9 \mathrm{fm}$ for $U$. Data show some erratic behavior around the average $A$ dependence. This is due to the fact that annihilation takes place from a bound Coulomb state, whose principal quantum number can jump from one value to the next when the target charge increases (see Refs. $[1,14]$ ).

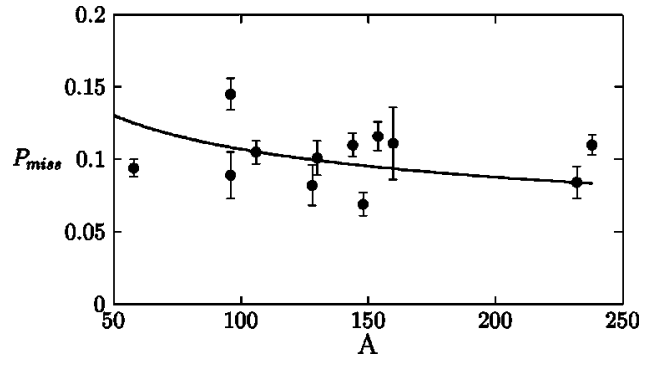

FIG. 4. Comparison between the experimental values (dots, Ref. [3]) for the relative probability for single-nucleon events and the fit with Eq. (12). See text for details.

We have shown that the average location of the annihilation site of antiprotons on nuclei at rest can be extracted from the pion multiplicity for the whole set of events and from the single nucleon event yield for this kind of events. Different modes of interaction and different target "active zones" are implied in these two observables. Pion multiplicity is dominated by pion absorption, to be considered as a volume effect. On the contrary, for single nucleon events, it is necessary that there is no pion-nucleus interaction at all. Due to the sizable total $\pi$-nucleon cross section, the target active zone in this case extends much farther than the half-density radius. These considerations are reflected by the two different values of $\delta$ obtained from the analysis of the two experiments.

We acknowledge the support from the Belgian (Région Wallonne)_-Polish Collaboration Agreement and from the Polish Committee for Scientific Research (Grant No. 2 P03B 048 15).
[1] P. Jasselette, J. Cugnon, and J. Vandermeulen, Nucl. Phys. A484, 542 (1988).

[2] D. Polster, D. Hilscher, H. Rossner, T. von Egidy, F.J. Hartmann, J. Hoffmann, W. Schmid, I.A. Pschenichnov, A.S. Iljinov, Ye.S. Golubeva, H. Machner, H.S. Pendl, A. Grochulska, J. Jastrzȩbski, W. Kurcewicz, P. Lubiński, J. Eades, and S. Neumaier, Phys. Rev. C 51, 1167 (1995).

[3] P. Lubiński, J. Jastrzȩbski, A. Grochulska, A. Stolarz, A. Trzcińska, W. Kurcewicz, F.J. Hartmann, W. Schmid, T. von Egidy, J. Skalski, R. Smolańczuk, S. Wycech, D. Hilscher, D. Polster, and H. Rossner, Phys. Rev. Lett. 73, 3199 (1994).

[4] J. Jastrzȩbski, P. Lubiński, and A. Trzcińska, Acta Phys. Pol. B 26, 527 (1995).

[5] P. Lubiński, J. Jastrzębski, A. Trzcińska, W. Kurcewicz, F.J. Hartmann, W. Schmid, T. von Egidy, R. Smolańczuk, and S. Wycech, Phys. Rev. C 57, 2962 (1998).

[6] J. Cugnon, P. Deneye, and J. Vandermeulen, Nucl. Phys. 500, 701 (1989)
[7] G.E. Brown and W. Weise, Phys. Rep. 22, 279 (1975).

[8] E. Hernández and E. Oset, Nucl. Phys. A455, 584 (1986).

[9] G. Bendiscioli and D. Kharzeev, Riv. Nuovo Cimento 17, 1 (1994).

[10] M.A. Preston and R.K. Bhaduri, Structure of the Nucleus (Addison-Wesley, Reading, MA, 1975), p. 100.

[11] J. Cugnon and J. Vandermeulen, Ann. Phys. (Paris) 14, 49 (1989).

[12] D. Ashery et al., Phys. Rev. C 23, 2173 (1981).

[13] D. Ashery and J.P. Schiffer, Annu. Rev. Nucl. Sci. 36, 207 (1986).

[14] S. Wycech, J. Skalski, R. Smolańczuk, J. Dobaczewski, and J.R. Rook, Phys. Rev. C 54, 1832 (1996).

[15] J. Jastrzȩbski et al., Nucl. Phys. A558, 405c (1993).

[16] J. Cugnon, University of Liège, internal report, 1995.

[17] S. Wycech and R. Smolańczuk, Few-Body Syst., Suppl. 99, 1 (1995). 